\section{AN ADDRESS \\ ON THE}

\section{EYE COMPLICATIONS. OF THE ACUTE SPECIFIC FEVERS.}

Delivered before the Thames Valley Branch of the British Medical Association on March 8th, 1899.

BY PERCY FLEMMING, F.R.C.S., Assistant Ophthalmic Surgeon, University College Hospital.

Eye troubles frequently arise in the course of the acute specific fevers or remain as sequelæ of them. I propose to consider some of these to-day, and in doing so I shall limit the term "acute specific fevers" to the various fevers that are commonly met with in general practice in this country, and shall not include any account of syphilis, tuberculosis, septicæmia, etc.

Etiology and Pathology.

And in the first place let us see how the eye may be affected - that is, what are the possible channels by which the virus may reach the eye. The simplest way is, of course, by direct inoculation. This may be accidental, as when a variolous or vaccine pustule is scratched by the finger, and the latter inadvertently then applied to the eye. Or it may be, as in facial erysipelas, by direct microbic invasion, the cocci advancing in the lymphatics of the skin, and so vltimately reaching the lids and conjunctiva; but this is not a very common occurrence. More commonly (as in measles) the conjunctiva becomes inflamed as part of the general catarrhal condition, or, as in diphtheria, the inflammation may spread by continuity from the nose to the conjunctiva by the nasal duct. Such modes of infection are easily understood, but complications of deep structures such as the optic nerve, the orbital tissue, and even the central nervous system are frequently observed, and it is important to investigate their mode of origin. According to current pathological doctrines, such conditions are brought about by the absorption of "toxins," the products of the specific organisms, the toxins picking out particular structures and acting on them. Such a selective action is seen in the action of certain drugs : thus arsenic produces conjunctivitis, and tobacco (nicotine) acts on the optic nerve, producing definite structural change, and belladonna, taken internally, acts on the pupil and ciliary muscle. But in addition to this explanation, another is to be found in the anatomical relations that exist between the orbit on the one hand, and the nasal fossæ (with their communicating air sinuses) on the other. Inflammation of the mucous membrane of the nose is common in several of the feversnotably influenza and measles-and such inflammation may spread to the frontal, ethmoidal, and sphenoidal sinuses, and from these in turn the orbital contents may become involved. Moreover, the optic foramen or canal is only separated by a very thin plate of bone (which may in some cases be perforated) from the cavity of the sphenoidal sinus, and it is easy to realise that inflammatory conditions may involve the nerve there, and set up a retro-ocular neuritis, and such a neuritis does occur as a sequela of the fevers.

An acute febrile illness seems to leave the patient in a condition in which he is more susceptible to microbic invasion, such susceptibility being perhaps connected with the lower temperature that so frequently remains after the subsidence of the pyrexia. The pulmonary complications so common after measles and whooping-cough are examples of this second infection; and, in the same way, conjunctivitis, corneal ulcers, and keratitis are common sequelæ of measles, scarlet fever, and whooping-cough ; and the analogy between them is well exemplified in a case of orbital cellulitis occurring with influenza, in which the diplococcus pneumoniæ was found in the pus. The neglect of these late inflammatory conditions, for the most part superficial in the first instance, leads to destructive inflammation of the deeper tissues of the eye, and it is amongst the class that attend the hospitals that these severe complications of the acute specific fevers are met with.

A hæmorrhagic tendency may be induced by the altered blood state attendant on an acute specific fever, and of course is the factor in hæmorrhagic small-pox. This tendency may bring about impairment of vision either by producing retinal hæmorrhage, or, possibly, atrophy of the optic nerve may follow a hæmorrhage into its sheath.

Further, the movements of the eyeballs are dependent for their exact co-ordination on the activity of special nerve centres, and these centres may suffer in the general exhaustion of the nervous system that so frequently follows an attack of fever; and if owing to the state of refraction of the eyes there is an undue call on this centre, the latter in its weakened condition may be unable to maintain proper convergence of the eyes and squint may result, without any definite muscular paralysis. (Priestley Smith.)

In discussing the possible modes in which the exanthemata may affect the eye, it is important to bear in mind the frequency with which otitis media complicates or follows measles, scarlet fever, and less commonly whooping-cough ; and a definite connection has been shown to exist between suppurative otitis and the eye condition known clinically as "pseudo-glioma." This term is applied to cases characterised by the presence of a yellowish white reflex in the vitreous, often simulating very closely the appearance given by true glioma. Pseudo-glioma is a clinical expression, and at least three distinct pathological conditions may give rise to this peculiar fundus reflex, but by far the most common of these three is a retinitis or choroiditis giving rise to inflammatory exudation into the vitreous. Ophthalmic surgeons usually see these cases late, and then an indefinite history of some acute febrile illness is obtained, or the child is said to have had convulsions, and other symptoms suggestive of meningitis. In two cases of pseudo-glioma in which post-mortem examinations were made, purulent otitis with basic meningitis involving the optic nerve was found; in each case the eye affected was on the side opposite to the ear affected. And in another recently reported case, pseudo-glioma was associated with otitis media, and symptoms pointing to basic meningitis; though unfortunately no post-mortem examination was obtained. Short of producing a retinitis intense enough to cause extensive exudation, otitis media may set up an acute optic neuritis, and possibly cause paralysis of some of the ocular nerves, especially the sixth.

Finally, it must be remembered that Bright's disease is a frequent camplication of some, and may occur in any, of the acute specifics, and the various retinal complications-neuroretinitis, hæmorrhages, etc.-of the renal disease may appear as sequelæ of the acute specific.

Such, then, are the various modes in which the eye may become affected. Another most important factor to be considered is the presence or not of the "strumous diathesis." Many of these complications and sequelæe are slight and insignificant in themselves; but if the child be strumous or illnourished, the inflammations are prone to persist and lead to more serious and deep-seated lesions, specimens of which I shall be able to show you.

We will now consider the individual fevers in detail, taking the more common ones first.

Measles.

Measles is accompanied by general catarrh, and in the days preceding the rash the eyes become red and inflamed, water freely, and the coagulated discharge adheres to the lashes. This catarrhal condition increases with the rash, reaching its height about the second day, and then gradually subsiding. But if the child be delicate or strumous, this conjunctivitis may persist for long, and associated with it is frequentry an inflammation of the lid margins, which, if allowed to persist, leads to loss of the lashes, a loss which can never be made good, and which entails constant liability to damage from dust particles, etc., getting on to the cornea. Again, in these strumous children the conjunctival inflammation frequently leads to, or is associated with, corneal ulcers (phlyctens), and if these be neglected, perforation of cornes may ensue with consequent cyclitis, and subsequent shrinkage of the eye, so called "phthisis bulbi," as you see in this specimen removed several years after the attack of measles which caused its loss. In other cases the eye may be lost from the formation of a dense corneal cicatrix, with or without a staphylomatous condition of the cornea.

It will be seen that treatment in the early stages of the inflammation is veryimportant. For the conjunctivitis simple [200n] 
warm boric acid lotion, with a little boric acid ointment along the edges of the lids to prevent them sticking, is all-sufficient. If the tears are profuse, hot, and soalding an alkaline lotion (bicarbonate of soda gr. $x$ ad $\xi j$ ) gives relief. When marginal blepharitis is present, the scabs should be diligently removed (a rather painful process), and dilute ammoniated mercury ointment applied. 'The removal of the scabs will be facilitated by using a borax lotion. Ulcers must be treated in the ordinary way, cleanliness being ensured by frequent boric acid foment ations, atropine drops, and a shade over both eyes, or dark glasses may be worn; and it is often best to get these children out of doors (provided the general condition permits), protected from the light by dark glasses, rather than to coddle them up indoors.

Of the rarer eye complications in measles, perhaps the most interesting is the occurrence of optic neuritis. There is no doubt now that this may occur in measles independent of any other complication. On the other hand, it is important to remember that meningitis may occur with or after measles, and the occurrence of optic neuritis in measles should rouse suspicion of the coexistence of this complication, and should lead to examination of the ears. Transient amblyopia is said to occur in convalescence without any ophthalmoscopic sign, analogous to the uræmic blindness, but quite independent of kidney disease ; this amblyopia may persist for some considerable time.

Suppuration in the orbit, perhaps limited to Tenon's capsule, may occur either while the fever is at its height, or as in a case I have seen, during convalescence. In this latter case two distinct abscesses formed, producing proptosis, etc. but after opening and draining them the globe returned to its normal situation, and no ill-effects remained.

Measles often reveals the existence of hypermetropia. The essential clinical factor in hypermetropia is that the eye has to use its aecommodative power for distant vision, and in consequence requires more than the normal amount of accommodation for near work. If the amount of hypermetropia be small and the person vigorous and strong, the extra muscular effort required to see clearly causes no inconvenience. But the exhaustion following an acute illness may render this extra work very fatiguing. Hence during convalescence from measles, etc., especially on return to school, such a patient may complain of difficulty in reading, the eyes soon becoming tired and words "running into one another." Actual paralysis of accommodation, if it occur at all, is very rare, but this sort of accommodative impairment is very common, and should, of course, be corrected by glasses, which may only be required temporarily, at the same time that attention is given to general health, tonics being indicated.

Whooping-Covgh.

Whooping-cough, which so frequently occurs about the same time as measles, has few eye complications. The mechanical congestion that results from the paroxysm may. lead to subconjunctival ecchymoses, which are of no importance. But the possibility of such a hæmorrhage taking place in the optic nerve sheath or retina must be thought of. Children convalescing from whooping-cough seem liable to corneal ulcers. It is convenient here to mention the frequency with which one is told that a squint-the ordinary /"cross-eye"was noticed after the child had had measles or whoopingeough. This may be a mere coincidence in some cases, but it is easy to see how the weakness resulting from a severe attack of measles or whooping-cough increases the difficulty which a hypermetropic child has of maintaining binocular vision when the refraction of the two eyes is different. And as has been indicated previously the co-ordinating centre may be weakened by the febrile attack.

On the other hand, the possibility of the squint being paralytic and not concomitant must be kept in mind in cases of whooping-cough, for hæmorrhages may occur in the sheath of the sixth nerve, though this is very rare.

\section{Epidemio Parotitis.}

Mumps need hardly be mentioned. 'Weakness of accommodation has been noted, and it is possible that the lachrymal gland may participate in the general glandular inflammation, and be accompanied by effusion into the orbital tissues, causing œdema of lids and conjunctiva, and congestion of optic nerve.
SCARLET FEver.

Scarlet fever is sometimes, though rarely, accompanied by a catarrhal conjunctivitis when the rash is at its height. Much more frequently a chronic persistent muco-purulent discharge is seen as a sequelas. This is best treated by astringents, painting the everted lids with weak nitrate of silver solution (gr. $\mathbf{x}$ ad $\xi j$ ) being most useful, the patient himself using sulphate of zinc lotion, or alum, if the cornea be sound. Ulceration of the cornea is a rarer complication; this may be followed by perforation or destructive irido-cyclitis. As in measles, accommodative asthenopia may show itself in convalescence if the patient be hypermetropic.

The renal affection which occurs during convalescence may in its turn be complicated with typical "albuminuric retinitis." This, of course, occurs late. It-is difficult to give a definite prognosis as regards vision in these cases. It depends mainly on the type of Bright's disease present, and, speaking generally, the prognosis is better than in "ordinary" albuminuric retinitis, but this merely means that the Bright's disease does not, as a rule, become chronic. In contrast with these cases some have been recorded in which, without any albuminnria, marked neuro-retinitis was present, which ultimately subsided, good vision remaining. Transitory blindness without apparent retinal change, and, as in measles, lasting usually longer than " uræmic amaurosis," may occur, and, of course, true uræmic blindness may develop. Otitio media, such a common sequela of scarlet fever, may set up. optic neuritis, or possibly a retinitis with exudation (pseudoglioma).

Trphoid Fever.

Eye complications in typhoid fever are not common. The prolonged fever with its consequent weakness may cause subsequent accommodative asthenopia, and occasionally true paralysis of accommodation may occur, associated with mydriasis, in this latter respect differing from the postdiphtheritic cases. Rarely the extrinsie muscles may be paralysed, but recently I have observed a case of ptosis. Keratitis, abscess of cornea, choroiditis may possibly be met with. The former is especially likely to occur in those cases associated with "coma vigil," the corneal trouble being in part due to desiccation and exposure. In such cases it may be necessary to protect the eyes with a pad and bandage. But the greatest interest perhaps attaches to changes observed in the optic nerve. Optic atrophy has been observed several times after typhoid, and recently I saw a patient with double optic atrophy (without signs of past neuritis), who stated that the failure of sight began during convalescence from a bad attack of typhoid. Such an atrophy may be the result of retro-ocular neuritis set up by a toxin, or by inflammation reaching the nerve in the optic canal through the nose and sphenoidal sinus. This latter explanation seems the more probable in those cases of unilateral optic atrophy; and inflammation of the nose and throat is not uncommon in typhoid. The occurrence of marked papillitis-as distinet from retro-ocular neuritis-is, in my experience, rare in typhoid. Its presence in an undoubted case of typhoid suggests the possibility of meningitis coexisting, and should always lead to an examination of the ears, as the state of the latter may be responsible for the condition. On the other hand, meningitis is said to be an exceedingly rare complication of typhoid ; and if the diagnosis of the case be doubtful, I believe the presence of well-marked papillitis is strongly in favour of the case being one of meningitis and not typhoid. Perhaps, however, a localised meningitis may not ke such an uncommon occurrence in typhoid after all.

Cases of embolism of the central artery of the retina during convalescence from typhoid have been reported; it is quite possible that the lesion in some of these cases may be thrombosis and not embolism, the ophthalmoscopic appearances being practically the same.

\section{DIPHTHERIA.}

Diphtheria may affect the eyes in several ways. On the one hand, various paralyses may remain as sequelæ; and, on the other' hand, the conjunctiva may be the seat of the actual diphtheritic process, either primary or by spread from the nose.

Of the sequelæ, actual paralysis of accommodation is fairly common, and this condition is unaccompanied by mydriasis. 
The subjects of this paralysis that are seen by ophthalmic surgeons are almost invariably hypermetropic; the reason of this is that a myope or emmetrope is much less likely to be inconvenienced by a loss of accummodation than is a hypermetrope and therefore does not complain. In looking for this condition, it is important to remember that the pupil continues usually to react to the mental effort of accommodation and to the act of convergence, although the ciliary muscle itself may be totally paralysed. The symptom usually noticed about four or six weeks after the onset of the illness is the inability to see near objects : for example, reading or writing, or threading a needle, are difficult or impossible, and if the patient be a child it is frequently said that he or she can see better "with the mother's or father's glasses." The initial diphtheritic inflammation may have bern slight; often a history of "sore throat" is all that can be obtained. The treatment here is threefold: First take the opportunity of the cycloplegia to work out the state of refraction, and order glasses accordingly (the latter will, of course, not enable the patient to read fuently, but will help him to do so). Next drop in eserine (gr.ij ad $\zeta$ ) or pilocarpin (gr. $\xi_{\text {ij ad }}$ ) three times a day. And finally, it must be remembered that the paralysis of accommodation is merely part of a general poisoning of the nervous system (including the cardiac centre), and this must be met by proper rest, cod-liver oil, iron, arsenic, etc.

Much less common than this paralysis of accommodation is the paralysis of some of the extrinsic muscles, most commonly the externl, leading to squint, diplopia, etc. The prognosis in both these cases of paralysis is very favourable. I have observed papillitis several times in patients recovering from diphtheria, and this may be followed by atrophy.

The lids may be the seat of the diphtheritic process, either primarily or by spread. This diphtheritic conjunctivitis is a very destructive inflammation as far as the eye is concerned. Its chief characteristics are: great brawniness and infiltration of the lids, making an inspection of the cornea difficult, the presence of a definite membrane on the conjunctiva which is incorporated with the lid, and cannot be easily detached, and scanty discharge. The early recognition of these cases is very important, as the cornea is soon involved, and may slough unless the treatment be prompt and energetic. Further, it is important to recognise their diphtheritic nature, because, if regarded as a purulent conjunctivitis and treated with silver nitrate, matters are made worse, the raw surface made by the caustic quickly becoming covered by new membrane. The essentials of treatment are (I) the use of antitoxin and the usual routine treatment of diphtheria, and (2) the frequent washing of the conjunctival sac with some antiseptic, of which quinine lotion (gr. iv ad $\xi_{j}$ with a minimal quantity of sulphuric acid) seems best. Under this treatment the membrane quickly disintegrates and separates by a process of suppuration.

The bacterial diagnosis of these cases is not altogether satisfactory-at least from the practical clinical point of view. That is to say, the Klebs-Loeffler bacillus may be found in cases of conjunctivitis which are not of a virulent or severe nature; and, on the other hand, as in a case in the hospital last year, it was difficult at first to demonstrate the organism, although the case presented all the typical features of a diphtheritic conjunctivitis, and later on the typical bacilli were demonstrated. Practically, from the point of view of prognosis and treatment, a case presenting the clinical features previously mentioned is diphtheritic; other cases of membranous conjunctivitis, in which the lids are not brawny nor the membrane adherent, are not so serious, even though the typical bacillus be present.

INFLUENZA.

Influenza resembles diphtheria in several respects : in both there is a marked local inflammation followed by equally marked constitutional symptoms due to absorption of toxic products; and it is not surprising to find that the eye complications, more especially the late ones, of influenza resemble those of diphtheria. In the acute stages there is marked conjunctivitis, and sometimes corneal ulceration occurs Facial herpes is not an infrequent accompaniment of influenza, and in some of these cases minute herpetic vesicles form on the cornea, giving rise to much irritation and photophobia; these cases in contrast to the corneal c.mplications 5 that appear with herpes zoster ophthalmicus tend to recover quickly, and the corneal lesions themselves are usually superficial. Fuchs's keratitis (a not very common form of corneal inflammation) is generally set up or follows a severe cold in the head, and hence may be seen with influenza. It is characterised by minute dotted opacities under the epithelium, with some slight ciliary and conjunctival redness; the latter is usually quite readily subdued, but the little dots often persist for months before they finally disappear. Pain on movement of the eyes is, of course, one of the well-recognised symptoms of influenza, and may be muscular or due to some orbital inflimmation. As sequelæ there are accommodative asthenopia, true paralysis of ciliary muscle, and occasionally paralysis of the extrinsic muscles, just as in diphtheria. The acute inflammation of the nose and neighbouring cavity explains the occurrence every now and again of retroocular neuritis and orbital cellulitis and abscess. The neuritis usually comes on in fourteen to twenty-one days, is accompanied by deep orbital pain, and frequently a centrāl scotoma may be found. As a rule the prognosis in such cases is good, but atrophy may appear later. Acute optic neuritis and papillitis also occur, these cases differing from those of retro-ocular neuritis in that all the signs of inflammation observed by the ophthalmoscope are much more marked.

Central retinitis and retino-choroiditis may occur after influenza. In one case I had examined the patient for glasses a week or two before the onset of influenza, and had noted that the fundi were normal. In the attack one eye was very painful, and subsequently during convalescence it was discovered that the sight in that eye was very defective. Ophthalmoscopically a patch of central retino-choroiditis was found, and this has remained in statu quo for several years. Some while since I saw a patient who presented a very
striking appearance. The left eye, he said, became "bad" five days before he came to me, about a week after an attack of influenza; two days later the eye was painful and the lids more or less completely closed. When I saw him there was such swelling of the lower conjunctiva that a fold of it projected between. the lids and was nipped by them. On separating the lids, the conjunctiva was seen to be slightly injected, the cornea was clear, the eyeball was a little proptosed, and outward movement rather limited. The condition rapidly improved under hot fomentations, leeching, and purgatives. This case is probably one of serous inflammation of Tenon's capsule-so-called "serous tenonitis"-a condition characterised by conjunctival œdema, slight proptosis, and limitation of movements as in this case, and influenza is recognised as one of the causes of this condition. An accumulation of fluid-perhaps mere œdema and not inflammatory exudation-in the capsule certainly explains the symptoms. And Fuchs has recorded a case of suppuration in Tenon's capsule occurring with influenza in which the abscess discharged anteriorly and the pus was found to contain the pneumococcus. Such a case as this would have the same relation to my case as an empyema has to a simple pleurisy. I have seen a case of panophthalmitis occur in the course of an attack of acute influenza, and on enucleation there was found to be much surrounding orbital inflammation; and I think it quite likely that the eye in this case became inflamed secondarily to the orbital inflammation or to a tenonitis.

Eye complications in small-pox used to be very common; now in their severer forms they are rare. And the beneficial action of vaccination is most strikingly seen in the prevention of blindness. At the time when small-pox was very widespread, about one-third of all cases of blindness were due to it; now it is a rarity to see an eye lost from small-pox. I have notes of three cases of blindness due to an antecedent attack of small-pox, and in each of those the patient had for some reason or other not been vaccinated. One of these cases is almost of the nature of a crucial experiment, and it seems worth relating in detail. The patient was aged 27 when I saw her, and had a dense scar (leucoma) on the right eye, and a less dense one on the left. She could see a little with the latter, but was quite blind in the right. She was born in Scot. land, and soon after, her birth the family removed to. London. and in this way her vaccination was forgotten. In the 1871 epidomic all the family (hersolf and three sisters) were 
attacked at the same time with small-pox, and treated together. The three vaccinated sisters had a very mild attack and were scarcely marked at all, whereas the unvaccinated one was not only' badly marked; but practically blinded. Here all the conditions were the same, with the one all-important exception of vaccination.

In any case of small-pox-"modified" or not-the eye requires careful attention. In the acute stage the lids may be swollen from the presence of the eruption on or near them, and this swelling may be so great as almost to prevent the lids being separated, and may be accompanied by conjunctivitis simple or purulent. It is important to endeavour to prevent the lids sticking to one another by using some simple ointment, and in all cases, whether the lids be affected or not, the conjunctival sac should be washed out daily with some simple lotion. This, however, owing to the swollen condition of the lids, may be almost impossible, and may necessitate division of the outer canthus. More rarely pustules may occur on the palpebral and ocular eonjunctiva, much smalle than the pustules on the skin causing a purulent conjunctivitis, and later blepharitis; with loss of lashes, trichiasis, etc. may be met with. Such pustules, however, never form on the cornea. Keratitis, which is the most important and common complication that oceurs in the modified cases seen in the present day, is not due to formation of pustules, but arises altogether independently, occurring after the twelfth day of the illness, usually later, when the rash is drying up, and is the result of secondary infection. The cases I have seen have been slight in severity, and have taken the form of superficial keratitis and vascular ulcer; in other cases the keratitis is severe, and runs on into abscess of cornea, which in turn may lead to perforation with prolapse of iris, etc. It is the scar resulting from this corneal ulceration, or the destruction of the eye following perforation, that causes the blindness. The treatment is mainly preventive-vaccination in the first place, and in the presence of an actual case of small-pox regular systematic examination of the eye, with the use of simple antiseptic lotions; and this examination may very likely be carried out less regularly just at the most important time, because the keratitis usually cornes on as the patients are getting better. If the ulcer has formed, treatment on general principles must be followed: atropine drops, perchloride of mercury, or quinine lotion in mild cases, and nitrate of silver or actual cautery applied to the ulcer if it show an infiltrated spreading edge. Bearing in mind too the fact that the eye is infected from the other pustules, it would seem an important part of treatment to endeavour to treat the pustules about the face with antiseptic applications.

The other complications of small-pox are not of special interest. Thus the various "strumous affections" are likely to develop after convalescence ; ordinary iritis may occur, as also paralysis of accommodation, and, rarely, albuminuric retinitis. A diffuse neuro-retinitis has been described, occurring at the time when the rash is drying. Inflammation of the lachrymal sac, and, later, stricture of the nasal duct, have been observed, and attributed to formation of pustules in the duct or nose.

\section{VACcination}

Vaccination may be followed by eye complications, either the patient himself directly inoculating his own eyelid or conjunctiva from a ruptured vesicle, or more commonly by a child inoculating its mother's or nurse's eye. In this way typical vaccine vesicles and pustules may develop on the lids, causing swelling and œdems, or a severe conjunctivitis may be set up characterised by much swelling of the conjunctiva (chemosis), which may be severe enough to produce corneal ulceration and hypopyon.

The treatment here is clearly to prevent infection of the conjunctiva if the pustule has developed on the lid, and if the sac is already, infected to empty the vesicle as soon as possible, foment the eye, and drop in atropine. . When such cases as these are seen without any previous history to guide one, the diagnosis has to be made from 'a primary syphilitic chancre; but the fact that the ulcer is clean cut, much indurated, and has taken a considerable time to develop; will all point to syphilis, and once the possibility of vaccination is thought of, the history will make the diagnosis.

VARICHLAA:

Várioella is sometimes accón paniod lby a mild conjuñctiv- itis. As rarities it may be noted that one case of double panophthalmitis has been recorded, and another of retro-ocular neuritis apparently due to chicken-pox, but possibly these may have been mere accidental as ociations.

Facial erysipelas may directly affect the eye. Most usually the lids become swollen and oedematous and cannot be separated; this swelling may completely subside without doing any damage, but the swelling may be so great that gangrene of the lids may result or suppuration take place. It becomes therefore very important to relieve this excessive distension of the lids by small punctures, and to be ready to make a free incision if distinct fluctuation can be obtained. Such an incision is best placed so as to lie in the fold of skin at the inferior border of the lower lid, and if the pus be early let out no deformity need result. On the other hand, when there is distinct gangrene and loss of substance, ectropion and all its consequences may ensue, and plastic operations will sub. sequently be required for the correction of the deformity. As in the other fevers the cornea may ulcerate and destructive inflammation of the eye result, and the swollen state of the lid makes it very difficult to recognise and treat such complications.

The inflammation of the subcutaneous tissues may extend into the orbit and involve the orbital tissues, producing "orbital cellulitis" or orbital abscess, with extreme exophthalmos, and the inflammatory process may here involve the sheaths of the optic nerve, giving rise to optic neuritis, or by compressing the vessels cause atrophy of the nerve. Possibly the inflammation may spread back and involve the meninges and venous sinuses, and by way of the circular sinus may reach the other orbit. Hence the early recognition of orbital cellulitis is most important, free incision and drainage being obviously indicated. In other cases the suppuration, instead of being in the general orbital cavity, may be limited to Tenon's capsule, and it is quite possible that the latter may in some cases be the starting point of the former. Obviously, if the pus be limited to the capsule, the prognosis is much more favourable, and early incision is very important. The fact that loss of sight may follow an attack of facial erysipelas seems very inadequately noted in general textbooks. It may be due, as above described, to an orbital cellulitis, but erysipelas may cause papillitis and retro-ocular neuritis without setting up orbital cellulitis, although the latter has been stated by some to be a necessary factor in their causation.

The prognosis as regards vision is, as a rule, much better in the cases of retro-ocular neuritis, even though visual acuity at one time may be reduced to mere perception of light. But subsequent atrophy may set in, and the patient may first be seen when the nerve is already atrophic.

Another cause of blindness or impairment of vision in erysipelas is hæmorrhagic retinitis due to thrombosis of the retinal veins; this may be the result of inflammation of the orbital tissue, and in one case the actual thrombosis was observed to take place two days after the extension of inflammation from the lids to the orbit.

Before leaving the subject of erysipelas it is worth while mentioning a point of importance with regard to diagnosis. Herpes zoster in the territory of the ophthalmic nerve is accompanied by redness, swelling, and vesication of skin, and quite frequently by ulceration of the cornea ; this condition, not being so well krown as facial erysipelas, is very commonly mistaken for the latter, but attention to the unilateral distribution of the herpetic eruption will prevent the mistake being made. Facial herpes, as has been mentioned, is a fairly frequent accompaniment of acute febrile illness, and associated with the herpetic vesicles on the face may be a crop of similar vesicles on the cornea, giving rise to much photophobia, lachrymation, etc., and requiring the eye to be protected by a pad and bandage.

AN Antirabic Instrtute at Hanol.-In accordánce with instructions issted by $M$. Guillain, French Minister of the Colonies, a serumtherapeutic department and an antirabic service have been established in connection with the hospital at Hanoi in Tonquin. Rabies is said to be very prevalent in Tonquin, and persons bitten by rabjd animals have hitherto had to be-sent to Saigon for treatment. 